Bibliotecas. Vol 36, N¹ Especial, 2018. EISSN: 1659-3286

URL: http://www.revistas.una.ac.cr/index.php/bibliotecas/index

DOI: http://dx.doi.org/10.15359/rb.36-1.4

Licencia: Creative Commons (BY-NC-ND) 4.0 Internacional

\title{
Perfil del docente de la Universidad Nacional Mayor de San Marcos y su pertinencia con las demandas actuales: Escuela Profesional de Bibliotecología y Ciencias de la Información
}

Profile of the University Professor of the National University of San Marcos and its Rertinence with the Current Demands: School Professional of Library and Information Science

Alonso Estrada-Cuzcano*, Universidad Nacional Mayor de San Marcos, Perú

Karen Lizeth Alfaro-Mendives* Universidad Nacional Mayor de San Marcos, Perú

\section{Resumen}

El presente trabajo pretende realizar una recopilación teórica y reflexiva acerca del perfil profesional del docente universitario, asimismo, se realiza un análisis de las competencias que debe exigir este perfil para que logre satisfacer las necesidades de la sociedad actualmente, teniendo en consideración los cambios tecnológicos y las nuevas demandas del mercado laboral, concluyendo que este perfil debe estar constantemente actualizándose para garantizar generaciones de profesionales idóneos y con valores que cumplan estas exigencias. Por último, se analiza el perfil de los docentes de la Escuela Profesional de Bibliotecología y Ciencias de la Información, identificando sus conocimientos, habilidades y experiencia profesional.

Palabras clave: Perfil profesional / docente universitario / bibliotecología. 


\begin{abstract}
s
This paper intends to make a theoretical and reflexive collection about the professional profile of the university professor, also an analysis of the competences that this profile is required to meet the needs of society today, taking into account the technological changes and the New demands of the labor market, concluding that this profile must be constantly updated to guarantee generations of qualified professionals with values that meet these requirements. Finally, the profile of the teachers of the Professional School of Library and Information Science is analyzed, identifying their knowledge, skills and professional experience.
\end{abstract}

Keywords: Professional profile / university teaching / librarian.

\title{
I. Introducción
}

Existen varios estudios sobre el perfil profesional del docente universitario, algunos basados en los cambios que la sociedad del conocimiento requiere (Bozu \& Canto, 2009; García, Reyes \& Pachano, 2012) y otros basados en las competencias (Mas, 2011; Álvarez, 2011; Rivera Michelena, N., 2016); sin embargo, es necesario establecer ciertos criterios para referirse al profesional docente. En primer lugar, este debería estar ajustado a las demandas que el mercado laboral exige y, en segundo lugar, considerar que este perfil debería estar basado en competencias, tomando en cuenta los nuevos paradigmas educativos.

Para ser más específicos, se define a la competencia como saber, saber hacer, poder hacer y querer hacer; según Mas (2011) el constructo competencia es un término polisémico, ambiguo, con diversidad de acepciones (según el referente cultural, el ámbito donde sea utilizado, entre otros) y complejo por los componentes que la integran, considerándose que las competencias son mucho más que una simple acumulación de contenidos (saber), están también constituidas por habilidades (saber hacer), actitudes y valores (saber ser y estar), adquiriéndose y/o desarrollándose mediante simulaciones formativas, a través de la propia 
experiencia social y laboral.

En consecuencia, las universidades se convierten en un centro privilegiado en donde con mayor frecuencia el equipo docente se encuentra con la tarea principal de profundizar en el análisis de los procesos y modelos educativos, así como también de plantear alternativas innovadoras $y$, en paralelo, evaluar su impacto social en búsqueda del desarrollo y consolidación de la institución educativa. Se puede argüir que las competencias involucran además de conocimientos y habilidades, valores y actitudes, factores indispensables en cualquier profesional.

\section{Entorno universitario peruano}

\section{a. La universidad peruana}

Es importante contextualizar a la universidad peruana dentro el marco jurídico bajo la cual se rige: Ley $\mathrm{N}^{\circ}$. 30220 denominada Ley Universitaria (Ley $\mathrm{N}^{\circ}$. 30220, 2014, julio 9). Esta ley tuvo muchas dificultades para su aplicación inmediata debido a una serie de demandas de inconstitucionalidad que finalmente fueron resueltas por el Tribunal Constitucional que ratificó la constitucionalidad de la ley en todos sus extremos (Tribunal Constitucional, 2015).

Señalaremos algunos apartados de la ley que se refieren a la labor docente. El art. $5^{\circ}$ de la ley reconoce, entre sus principios, la libertad de cátedra, la autonomía, el espíritu crítico y de investigación; así como, el pluralismo, tolerancia, diálogo intercultural e inclusión, el mejoramiento continuo de la calidad académica y la creatividad e innovación (Ley № 30220 , 2014, julio 9). Se puede afirmar que todos estos principios que rigen en la universidad se enmarcan en la labor y en el perfil del docente.

Es imprescindible referirse al régimen de estudios que se aplica a la universidad peruana; según la ley (Art. 31) las universidades establecen su régimen académico por Facultades y 
estas pueden comprender: los Departamentos Académicos que nuclean a los docentes; las Escuelas Profesionales que son responsables de los estudiantes y administra los recursos de la escuela; las Unidades de Investigación que coordinan actividades con los institutos de investigación, los grupos de investigación y además realizan un seguimiento de los fondos concursables; y las Unidades de Posgrado encargadas de las maestrías y doctorados (Ley №. 30220, 2014, julio 9).

En cuanto a los docentes universitarios la ley (Art. 79) señala como funciones: la investigación, el mejoramiento continuo y permanente de la enseñanza, la proyección social y la gestión universitaria, en los ámbitos que les corresponde (Ley №. 30220, 2014, julio 9). Los docentes, según la ley (Art. 80), son: ordinarios: principales, asociados y auxiliares; además, extraordinarios: eméritos, honorarios y similares dignidades que señale cada universidad y que no podrán superar el $10 \%$ del número total de docentes que dictan en el respectivo semestre (Ley №. 30220, 2014, julio 9). Así mismo, el régimen de dedicación de los docentes ordinarios (Art. 85) en dedicación exclusiva (única actividad remunerada); a tiempo completo (40 horas semanales) y a tiempo parcial (menos de cuarenta (40) horas semanales) (Ley $\mathrm{N}^{\circ}$. 30220, 2014, julio 9).

La ley (Art. 86) introduce la figura del docente investigador que es designado en razón de su excelencia académica y su carga lectiva será de un curso por año; además tiene una bonificación especial del $50 \%$ de sus haberes totales, según estándares del Sistema Nacional de Ciencia, Tecnología e Innovación Tecnológica (SINACYT) (Ley №. 30220, 2014, julio 9).

\section{b. Modelo Educativo San Marcos}

El Modelo Educativo San Marcos señala las líneas directrices para la formación académica y es un documento que sirve de guía para el desarrollo de los planes de estudio que elaboran o diseñan las diferentes Escuelas Profesionales de la universidad.

El Modelo Educativo San Marcos integra los siguientes ejes transversales:

- Aprendizaje centrado en el estudiante 
- Educación a lo largo de toda la vida

- Formación integral basada en competencias

- Ética

- Investigación

- Innovación educativa

- Responsabilidad social universitaria

- Interculturalidad

- Transdisciplinariedad

- Cultura de calidad educativa (UNMSM, 2015, p. 29).

E integra esas características generales al currículo de San Marcos, el cual está delineado como:

- Innovador; tendencia referida a la modificación e introducción de nuevos métodos y estrategias.

- Prospectivo; se refiere a la formación de los estudiantes con capacidad de previsión, anticipación y adaptación respecto al futuro.

- Integrador; busca convertir en transversales los ejes educativos como la investigación, la educación en valores, el 'aprendizaje-servicio solidario' y protección al medio ambiente para incorporarse a las actividades propias de cada carrera.

- Humanístico; enfatiza el respeto a las personas, la dignidad y valores. Resalta aquellas manifestaciones que permite al ser humano trascender, como el arte, la ciencia, la cultura, para crear una cultura de paz

- Flexible; permite al estudiante la libertad de elegir asignaturas que complementen su formación académica, en la propia universidad u otras (UNMSM, 2015, pp. 41-42).

El Modelo San Marcos establece algunos lineamientos generales para la construcción de un plan de estudios, este documento también delinea el papel del docente sanmarquino:

- Acompaña y orienta al estudiante para su desarrollo personal y consecuentemente su buen desempeño académico. 
- Prioriza el aprendizaje para el logro de las capacidades del estudiante.

- Realiza investigación y orienta el proceso de producción, aplicación y difusión de nuevos saberes.

- Promueve el aprendizaje-servicio solidario.

- Se desempeña con ética, sentido crítico y autocrítico y responsabilidad profesional.

- Promueve la investigación formativa (UNMSM, 2015, p. 46).

Mientras que el perfil del docente sanmarquino, según el Modelo San Marcos, contempla los siguientes aspectos:

1. Compromiso ético.

2. Domina su especialidad, se actualiza y capacita.

3. Aplica estrategias innovadoras para el aprendizaje.

4. Diseña y ejecuta proyectos de investigación.

5. Formación integral (científica, humanística, tecnológica y social).

6. Capacidad de trabajar en equipo.

7. Capacidad de análisis y síntesis.

8. Capacidad crítica y autocrítica.

9. Liderazgo.

10. Dominio de la comunicación oral y escrita.

11. Capacidad tutorial.

12. Conocimiento de una segunda lengua.

13. Dominio de las nuevas tecnologías de información y comunicación (NTIC).

14. Innovador, creativo y emprendedor.

15. Comprometido con el desarrollo sostenible y racional (UNMSM, 2015, p. 47)

El Modelo Educativo San Marcos es considerado clave para la definición, funciones, fines y principios establecidos en el Estatuto que rige en la actividad académica y administrativa de la universidad (Resolución Rectoral N. 0313-R-16, 2016, agosto 17). 


\section{c. La Bibliotecología y Ciencias de la Información en la UNMSM}

Se toma como referencia el plan de estudios recientemente aprobado (UNMSM, 2016) con el cual se puede evidenciar una evolución de la profesión a partir de un marco legal:

- Creación de la Escuela Nacional de Bibliotecarios - ENB. Resolución Suprema del 23 de junio de 1943.

- Ley que establece el Día del Bibliotecario, Ley № 16801 del 9 de enero de 1968.

- Aprobación del Convenio de transferencia de la ex Escuela Nacional de Bibliotecarios ENB a la Universidad Nacional Mayor de San Marcos - UNMSM, Resolución Rectoral № 60385 del 21 de marzo de 1980.

- Convenio entre la UNMSM y el Instituto Nacional de Cultura para la transferencia de la ex Escuela Nacional de Bibliotecarios del Instituto Nacional de Cultural a la UNMSM, del 10 de abril de 1980.

- Crean el Colegio de Bibliotecólogos del Perú como institución autónoma, Ley № 25189, de 18 de enero de 1990.

- Estatuto del Colegio Profesional de Bibliotecólogos.

- Ley № 29181, Ley del Ejercicio Profesional en Bibliotecología y Ciencias de la Información, del 10 de enero de 2008 (UNMSM, 2016, pp. 8-9).

La creación de la Escuela Nacional de Bibliotecarios - ENB se produce a raíz del incendio de la Biblioteca Nacional del Perú - BNP, un acontecimiento nefasto para el país; los bibliotecarios se encargarían de reconstruir la biblioteca destruida por el fuego. LA ENB permanecería bajo la administración de la BNP hasta 1980 cuando se produce un convenio que permite el traslado de la ENB a la UNMSM donde funge hasta el día de hoy.

Se incluyen en este marco jurídico algunas leyes que promulgó el Congreso en las que se aprueba una fecha para celebrar el día del bibliotecario; la creación del Colegio de Bibliotecólogos del Perú y la Ley del Ejercicio Profesional en Bibliotecología y Ciencias de la Información; y se desconoce el impacto de estas leyes en el mercado laboral, especialmente 
en el sector público que está obligado a la contratación de bibliotecarios profesionales en los diferentes tipos de bibliotecas.

Existen algunos trabajos de investigación que acercan un poco a la historia y evolución de la Bibliotecología en el país y en la UNMSM, que se citarán a continuación. El fundador de la ENB plasma en sus memorias las dificultades que pasó para la creación de la citada escuela (Basadre, J., 1975); otras autoras hacen un balance de la formación en la ENB y realizan algunas reflexiones históricas de su trascendencia (Mac Kee de Maurial, N., 1963; Mac Kee de Maurial, N., 1966; Pardo Sandoval, T., 1990). Existen trabajos globalizadores que involucran la historia de la enseñanza de la Bibliotecología sin distinción de las instituciones académicas, sino como un corpus nacional (Corzo, O., \& Lumbreras, S., 2003; TalaveraIbarra, A. \& Vega-Ramírez, A. d. I., 2016). Sin embargo, hay otros trabajos que reseñan la situación de la Escuela Profesional de Bibliotecología de la UNMSM en determinados periodos (Miranda Meruvia, I., 2005; Estrada-Cuzcano, A. \& Alfaro-Mendives, K. L., 2016); los trabajos reseñados aquí son muy significativos y pueden mostrar un panorama completo de la evolución histórica de la Bibliotecología en el Perú.

Actualmente, la Escuela Profesional Bibliotecología y Ciencias de la Información propone lo siguiente:

Misión: "Somos la escuela académica que forma profesionales en Bibliotecología y Ciencias de la Información altamente calificados, orientados a la investigación, con valores éticos, conocimientos científicos y tecnológicos en el acopio, procesamiento, análisis, acceso, difusión y gestión de la información, que contribuyan al desarrollo científico y cultural de nuestra sociedad".

Visión: "Ser la Escuela líder en la formación de profesionales con excelencia académica en el campo de la Bibliotecología y Ciencias de la Información a nivel nacional e internacional, que responda a los retos planteados en la sociedad, impulsando la investigación y estrechando vínculos con los diferentes sectores sociales y culturales como elemento clave en la gestión de la información y conocimiento" (UNMSM, 2016, p.10). 
Sin embargo, no existe un rol y perfil del docente que dé lugar a realizar esta investigación para obtener algunos datos relevantes y establecer una especie de estado del arte de la función docente en la escuela.

\section{Objetivos y metodología}

\section{a. Objetivos}

- Realizar una revisión teórica y reflexiva acerca del perfil profesional que debe tener el docente universitario para satisfacer las necesidades de las demandas actuales.

- Determinar el vacío que existe entre el perfil actual de los docentes y lo que demanda el contexto laboral actual.

- Realizar un análisis del perfil profesional de los docentes de la Escuela Académico Profesional de Bibliotecología y Ciencias de la Información.

\section{b. Metodología}

La presente investigación es de tipo básica, nivel descriptivo simple, diseño no experimental transversal, de enfoque cuantitativo y cualitativo, bajo la modalidad de estudio de casos. La técnica empleada es la revisión documental y el instrumento, la ficha de registro. La muestra estuvo constituida por 15 docentes.

\section{Perfil profesional}

Es claro para todos que cuando se habla de perfil profesional se está refiriendo al conjunto de competencias que debe tener una persona para poder desenvolverse con éxito en un puesto determinado, considerando no solo conocimientos, sino también habilidades, valores y actitudes y que estás estén alineadas con lo que se necesita en el mercado laboral. Así Jaramillo (2015) entiende como perfil profesional:

Lo que se debe saber para ser y ejercer, es decir, la adquisición de los saberes, destrezas y habilidades para el desempeño profesional y la adaptación a las tendencias 
de la recuperación, organización, tratamiento, gestión y difusión de la información, mientras que perfil laboral corresponde a los saberes, destrezas y habilidades que se adquieren después de graduarse en la universidad, este perfil es adquirido por la experiencia de empleabilidad, los cambios que exige el entorno donde se labora, por las condiciones de adaptabilidad, flexibilidad y por habilidades para solucionar problemas. (p. 113)

En el caso puntual de un docente universitario, este perfil tiene que estar por demás consolidado, pues es aquel que forma a los futuros profesionales que van a ocupar un lugar dentro del mercado laboral en el futuro. Segura (2004, pp. 19-20), citado por Álvarez (2011) menciona que:

(...) pensar en el perfil integral del profesor universitario que puede concebirse como el conjunto organizado y coherente de atributos o características altamente deseables en un educador, que se materializan en los conocimientos que posee, las destrezas que muestra, las actitudes que asume y los valores que enriquecen su vida personal y educativa, por ende, a sinergia de este conjunto de atributos le permitirá desempeñarse eficientemente, con sentido creador y crítico, en las funciones de docencia, investigación/creación, extensión y servicio que corresponden a su condición académica, concebidas como funciones interdependientes, comprometidas en el logro de la misión de la universidad. (p.105)

La universidad, lugar donde el profesor universitario desarrolla su trabajo, está en uno de los mayores momentos de transformación de su historia, estos profundos cambios por la calidad y la internacionalización que está viviendo la universidad conllevan alteraciones en las funciones, roles y tareas asignadas al profesor, exigiéndole a este el desarrollo de nuevas competencias para desarrollar adecuadamente sus funciones profesionales (Mas, 2011).

Álvarez (2011) sugirió el siguiente perfil profesional para el docente universitario:

- Habilidades de gestión: vinculadas a la gestión, organización y planificación eficiente de la enseñanza y de sus recursos en diferentes contextos. 
- Función de tutor o tutora: ya que orienta en el autoaprendizaje del estudiantado, creando un ambiente propicio para el aprendizaje individual y colectivo.

- Capacidades culturales y contextuales: resultando imprescindible poseer una cultura general, conocer al estudiantado y los productos culturales con los que se relaciona.

- Capacidades comunicativas: vinculadas a la capacidad discursiva, o sea, a la posibilidad de apropiarse de diferentes recursos del lenguaje a nivel verbal y no verbal, los cuales le permitan transmitir al docente experiencias y provocar aprendizajes. Favorece la comunicación docente-estudiante y permite establecer un diálogo con los contextos. Se trata, pues, de una comunicación cuidadosamente elaborada, sin descuidos, rigurosa, clara, bien estructurada y, especialmente, dirigida al interlocutor.

- Capacidades sociales: relacionadas con acciones de relación social y colaboración con otras personas, el trabajo en equipo y el liderazgo para favorecer el interaprendizaje entre docentes y entre docentes y estudiantes.

- Capacidades metacognitivas: (...) relacionadas con la capacidad crítica, autocrítica y reflexiva del docente con el objetivo de que este sea capaz de revisar su actuación docente y mejorarla de forma sistemática, así como la capacidad de reacción ante situaciones conflictivas, novedosas o imprevistas, la creatividad y la innovación didáctica y la toma de decisiones mediante la previa identificación del problema, recopilación de toda la información y propuesta de soluciones.

- Capacidades tecnológicas: relacionadas con el aprendizaje, la investigación y el uso de las posibilidades que las tecnologías de la información y la comunicación brindan a la labor profesional docente. Pone énfasis en una reflexión profunda que le posibilite al docente realizar una mediación pedagógica de estas y, de esa forma, en los procesos de búsqueda, selección y síntesis de la información, establecer puentes entre ellas y el estudiantado.

- Características de investigación: estas les permitan a los docentes: a) Construir proyectos futuros integrales que ubiquen y motiven el quehacer de los alumnos en este mundo, con una concepción de lo que es el ser humano, sus posibilidades y trascendencia, b). Buscar nuevas metodologías, información y recursos para su propia 
formación y la del alumnado y c). Enseñar a pensar, a descubrir, a formular y a buscar (Ortega, 2010, p. 318, citado por Álvarez, 2011).

Además de las características mencionadas que debería tener el docente universitario, se considera que, sobre todo, debe poseer ciertas cualidades que lo caractericen como un ser humano capaz de emprender la difícil y gratificante tarea de la docencia, entre las que destacan: honestidad, principios éticos y sensibilidad con el otro.

En cuanto a los conocimientos que debe manejar el profesional de la información para atender las nuevas demandas, se encuentran la pedagogía, las tecnologías para la investigación, los costos y los presupuestos, marketing, métricas de investigación, social media management, entre otros. Jaramillo (2015) afirmó que en la voz de egresados y empleadores es fundamental para los profesionales de la información, fortalecer las áreas de TIC, Administración, Análisis y Organización de la Información, Servicios, Gestión Documental y en aspectos relacionados con la investigación y la docencia.

Jaramillo (2013), citado por Jaramillo (2015), mencionó que los perfiles de los profesionales de la información se han definido a partir de dos vertientes. Una, desde la fundamentación y principios de la profesión y, la otra, desde las demandas del mercado laboral. Es así como las asociaciones e instituciones relacionadas con la formación profesional han contribuido en este aspecto con trabajos y aportes de instituciones como la American Library Association-ALA, Euroguide LIS y la International Federation Library Organization -IFLA- (Euroguide LIS, 2004), quienes plantean las áreas fundamentales de dominio del profesional de la información. Es así que en Colombia se encontraron contenidos esenciales para la formación en siete áreas: Fundamentación de la Información, Caracterización de la Información, Recursos y Fuentes de Información, Servicios de Información, Organización y Tratamiento de la Información, Gerencia y Generación de Información, Comunicación y uso la Información; áreas que son transversalizadas por las tecnologías de la información y la formación sociohumanística; contenidos que no distan de las definidas por los organismos internacionales. 


\section{Perfil profesional del docente en Bibliotecología y Ciencias de la Información}

En la actualidad la Escuela de Bibliotecología cuenta con 15 docentes. De ellos 5 son doctores, 3 maestros y 7 bachilleres, es decir cuentan con los grados necesarios según su especialidad y las asignaturas que enseñan. Asimismo, con las habilidades y actitudes necesarias para la ejecución del plan de estudios, ya que cada docente es especialista en las materias que imparte.

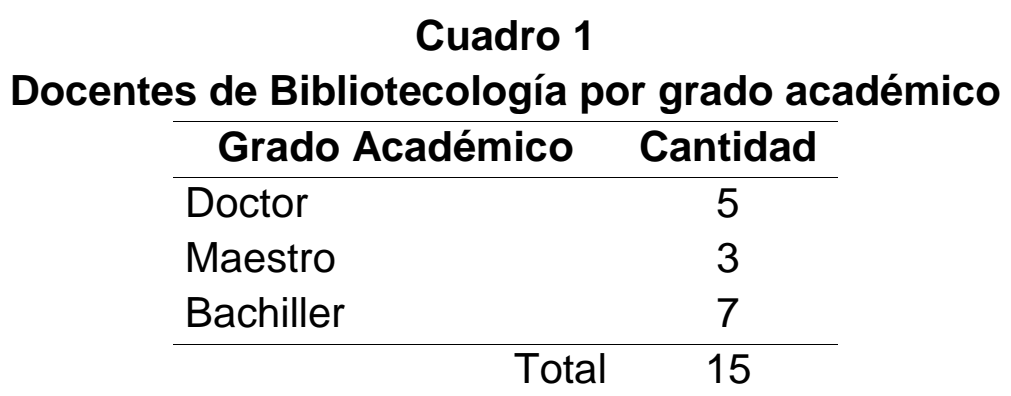

Fuente: Elaboración propia.

De los 15 docentes, 4 se encuentran registrados en el Registro Nacional de Investigadores en Ciencia y Tecnología. Es el registro de personas naturales que poseen capacidades establecidas de acuerdo con una calificación para realizar labores de investigación científica y/o desarrollo tecnológico (Concytec, 2017).

\section{Cuadro 2}

Docentes registrados en REGINA

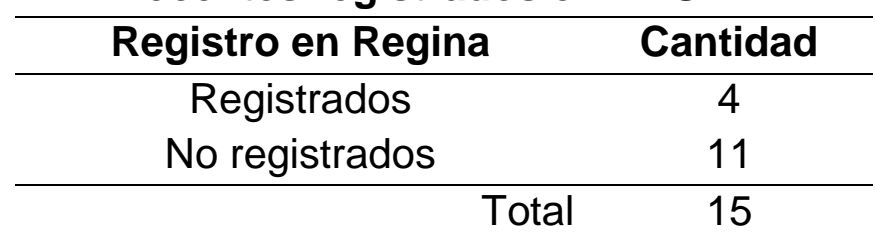

Fuente: Elaboración propia.

En lo que se refiere a su especialización, de los 15 docentes, solamente 5 se han especializado en la carrera propiamente dicha, sin embargo, los 11 restantes a través de cursos de perfeccionamiento, maestría y doctorados, así como su actividad laboral, han complementado la Bibliotecología con la Administración (3), Gestión de la información y el 
conocimiento (2) y en Tecnología, Estadística, Educación, Derecho y Archivística (1).

\section{Gráfico 1}

Especialización de los docentes

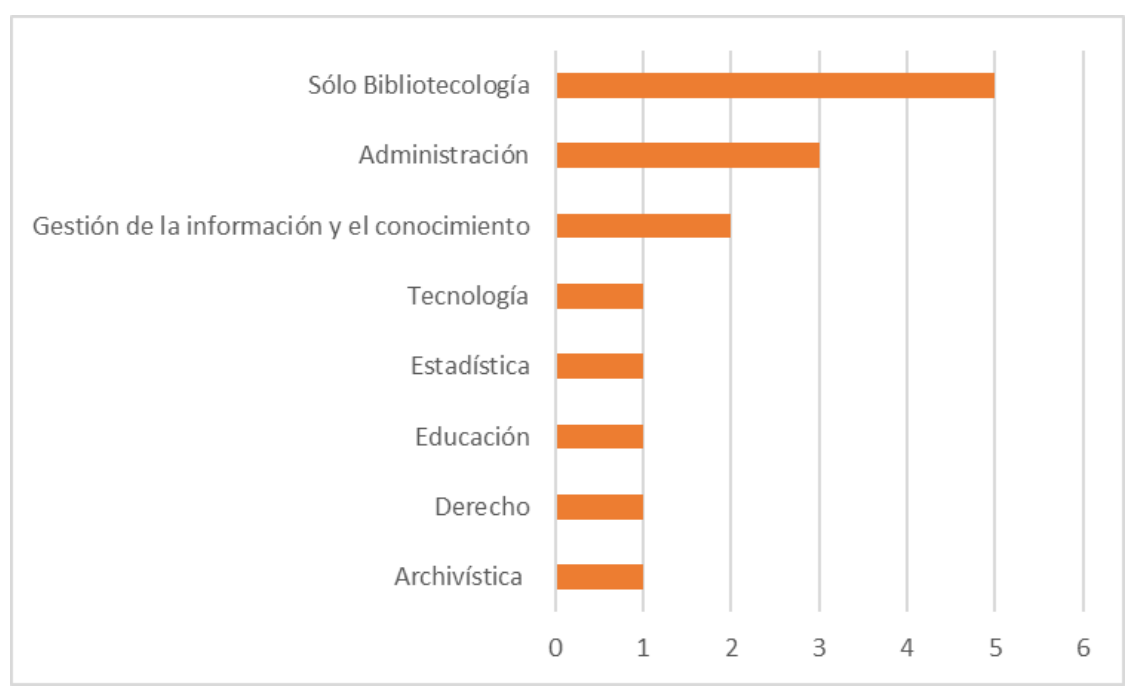

Fuente: Elaboración propia.

En lo que respecta al nivel de programa de estudio en los que imparte clases el docente, se puede concluir que los 15 docentes de Bibliotecología dictan en el pregrado en más de una universidad inclusive, solo 2 en maestría, inclusive en otras universidades también, sin embrago, ninguno de los docentes ha llegado a dictar en el doctorado.

En cuanto a la cantidad de años de experiencia en la enseñanza en la Escuela de Bibliotecología, entre los 15 docentes existe un promedio de 19 años en el ejercicio de la docencia, siendo el rango de cantidad de años entre 4 y 36 años.

\section{Cuadro 3}

\section{Nivel de programa de estudio en los que da clases el docente}

\begin{tabular}{lc}
\hline \multicolumn{1}{c}{ Niveles } & Cantidad \\
\hline Pregrado & 15 \\
Maestría & 2 \\
Doctorado & 0 \\
\hline
\end{tabular}

Fuente: Elaboración propia. 


\section{Gráfico 2}

\section{Cantidad de años en la Docencia en Bibliotecología}

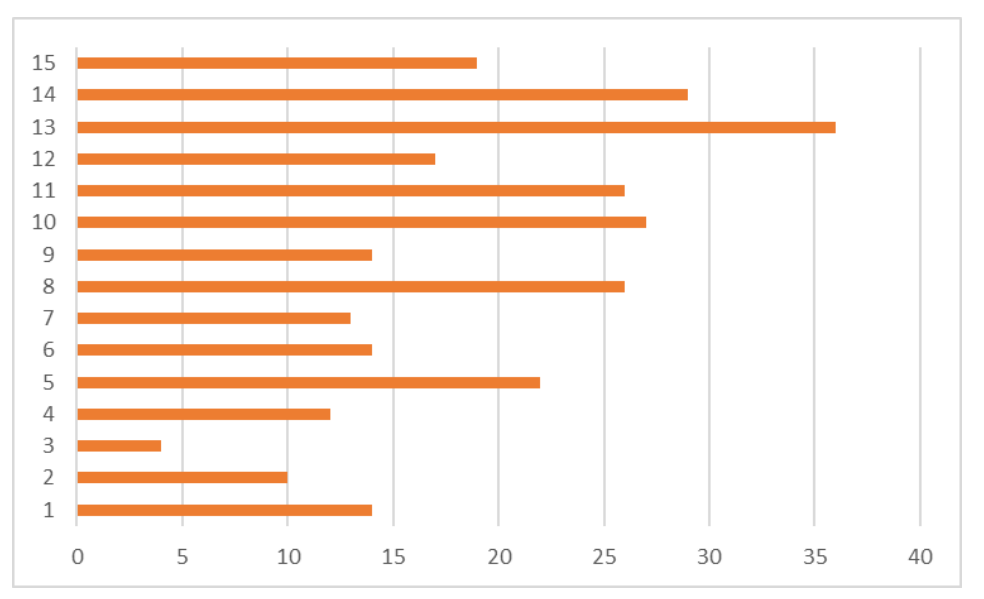

Fuente: Elaboración propia.

Con respecto a la categoría docente, de los 15 docentes, 2 son principales, 6 asociados y 7 auxiliares. Esto nos demuestra que la planta docente de la Escuela de Bibliotecología se encuentra en proceso de ascenso.

En lo que concierne al régimen de dedicación, de los 15 docentes, 4 son de dedicación exclusiva, 6 a tiempo completo y 5 a tiempo parcial, este dato es importante, ya que indica que existe una importante cantidad de docentes que están a dedicación exclusiva y tiempo completo, lo que muestra que tienen tiempo para la investigación, así como para la labor administrativa. Por otro lado, 5 de los docentes tienen tiempo parcial porque simultáneamente realizan labores diferentes a la docencia.

\section{Cuadro 4}

\section{Categoría docente}

\begin{tabular}{cc}
\hline Categoría & Cantidad \\
\hline Ordinario principal & 2 \\
Ordinario asociado & 6 \\
Ordinario auxiliar & 7 \\
\hline Total & 15 \\
Fuente: Elaboración propia.
\end{tabular}




\section{Cuadro 5}

Categoría docente

\begin{tabular}{lc}
\hline Régimen de Dedicación & Cantidad \\
\hline Dedicación Exclusiva & 4 \\
Tiempo Completo & 6 \\
Tiempo Parcial & 5 \\
\hline \multicolumn{1}{c}{ Total } & 15
\end{tabular}

Fuente: elaboración propia de los autores.

Como se puede observar, de la muestra analizada, existe una multidisciplinariedad importante en los docentes de la Escuela de Bibliotecología, existe gran potencial en investigación, como una de las principales habilidades del grupo, evidenciándose en el porcentaje de docentes inscritos en Regina. Cabe mencionar que la característica de investigador ha puesto de relevancia que la gran importancia del perfil profesional, estaría en la creación de conocimiento innovador, productivo, donde transmitir lo último sería verdaderamente lo importante y significativo en el rol del profesorado; la investigación sería la "niña bonita" y la docencia la "carga" (Mas, 2011, p.90).

Por otro lado, tienen muchos años de experiencia en la docencia, lo que se contradice con el poco número de docentes que ha alcanzado el nivel de docentes principales. Además de Bibliotecología, sus conocimientos son variados, especializándose en otras disciplinas como la administración, tecnología, educación, derecho, gestión del conocimiento y archivística. Asimismo, es importante mencionar que no han llegado a dictar en el nivel doctoral, siendo mínimo la participación en el dictado de maestría, esto último se justifica en la medida en que no existía, hasta el año pasado, maestría y doctorado en Bibliotecología, aspecto que se ha corregido, pues en el presente año, la Escuela de Postgrado de la Facultad de Letras y Ciencias Humanas se encuentra implementando una maestría en la especialidad.

La Escuela Profesional de Bibliotecología y Ciencias de la Información ha actualizado recientemente su plan curricular (UNMSM, 2016) y se mantiene en un continuo proceso de revisión, contribuyendo a que los estudiantes adquieran las competencias necesarias que 
exige la sociedad y, por ende, para cumplir con este objetivo es necesario docentes con un perfil competente y especializado necesarios para una acreditación.

\section{Conclusiones}

- El perfil profesional del docente universitario, basado en competencias, es la mejor elección que permite obtener cambios sustanciales en el aprendizaje y, por ende, garantizar un buen desenvolvimiento en el mercado laboral en el futuro, sin embargo, esto requiere un conjunto de adecuaciones, teniendo en consideración que el docente es uno de los pilares de la educación.

- La UNMSM tiene un modelo educativo que orienta la elaboración y el diseño de los planes de estudio que incluyen el rol y el perfil del docente en todas las escuelas profesionales.

\section{Referencias Bibliográficas}

Álvarez, M. (2011). Perfil del docente en el enfoque basado en competencias. Educare, 15 (1). Recuperado de http://www.redalyc.org/pdf/1941/194118804008.pdf.

Basadre, J. (1975). Recuerdos de un bibliotecario peruano: 1919-1930, 1930-1932, 1935 1942, 1943-1948, 1956-1958. Lima: Historia.

Bozu, Z. \& Canto, P. J. (2009). El profesorado universitario en la sociedad del conocimiento: competencias profesionales docentes. Revista de Formación e Innovación Educativa $\begin{array}{llll}\text { Universitaria. } & 2 & \text { (2). Recuperado de }\end{array}$ http://educacioncientifica.org/Refiedu/Vol2 2/REFIEDU 22 4.pdf

Corzo, O. \& Lumbreras, S. (2003). 50 años de enseñanza bibliotecológica 1943-1993. Lima: UNMSM.

Estrada-Cuzcano, A. \& Alfaro-Mendives, K. I. (2016). Presencia de la Universidad Nacional Mayor de San Marcos en la Bibliotecología Peruana: Estudio historiográfico. Letras, 87 (125). Recuperado de http://www.scielo.org.pe/pdf/letras/v87n125/a06v87n125.pdf 
CONCYTEC (2017). Guía para calificarte como investigador CONCYTEC. Recuperado de https://dina.concytec.gob.pe/appDirectorioCTI/index guiaRegina.jsp

García, C., Reyes, M. \& Pachano (2012). El perfil del docente universitario en el marco de la innovación curricular en Venezuela. Redhecs, 12 (7). Recuperado de http://publicaciones.urbe.edu/index.php/REDHECS/article/viewArticle/741/3010

Jaramillo, O. (2015).Pertinencia del perfil de los profesionales de la información con las demandas del mercado laboral. Revista Interamericana de Bibliotecología, 2 (38). ISSN 0120-0976. http://dx.doi.org/10.17533/udea.rib.v38n2a03

Ley № 30220 (2014, julio 9). Ley Universitaria. El Peruano Diario Oficial, Normas Legales.

Mac Kee de Maurial, N. (1963). Veinte años de existencia de la Escuela Nacional de Bibliotecarios. Boletín de la Biblioteca Nacional, 17 (28).

Mac Kee de Maurial, N. (1966). Escuela Nacional de Bibliotecarios del Perú. Fenix: Revista de la Biblioteca Nacional del Perú. (16).

Mas, Ò. (2011). El profesor universitario: sus competencias y formación. Profesorado: revista de curriculum y formación del profesorado, 15 (3). Recuperado de http://www.ugr.es/ recfpro/rev153COL1.pdf

Miranda, I. (2005). La Escuela Académico-Profesional de Bibliotecología y Ciencias de la Información de la Universidad Nacional Mayor de San Marcos, Perú: recuento y perspectivas. En F. F. Martínez Arellano \& J. J. Calva González (Eds.), Seminario INFOBILA: como apoyo a la investigación y educación en Bibliotecología en América Latina y el Caribe. Memoria 16, 17 y 18 de marzo de 2005. México: UNAM. CUIB.

Pardo, T. (1990). El primer cuarto de siglo de la Escuela Nacional de Bibliotecarios. Fenix: Revista de la Biblioteca Nacional, (36-37).

Resolución Rectoral Nº. 0313-R-16 (2016, agosto 17). Estatuto de la Universidad Nacional Mayor de San Marcos. El Peruano Diario Oficial, Normas Legales.

Rivera, N. (2016). Las capacidades humanas desde la perspectiva personológica del profesional competente. Revista Cubana De Educación Médica Superior, 30 (4). Recuperado de http://scielo.sld.cu/pdf/ems/v30n4/ems15416.pdf

Talavera-Ibarra, A. \& Vega-Ramírez, A. (2016). Library Education in Peru: Historical and Future Perspectives. In M. Seadle, C. M. Chu, U. Stöckel \& B. Crumpton (Eds.), 
Educating the Profession: 40 years of the IFLA Section on Education and Training. Berlin: Walter de Gruyter GmbH \& Co KG.

Tribunal Constitucional (2015). Sentencia del Tribunal Constitucional (Pleno) Nº. 0014-2014$\mathrm{Pl} / \mathrm{TC}$ de 10 de noviembre.

Universidad Nacional Mayor de San Marcos - UNMSM (2015). Modelo Educativo San Marcos [2… Ed.]. Lima, UNMSM. Vicerrectorado Académico. Recuperado en http://viceacademico.unmsm.edu.pe/wpcontent/uploads/2015/07/Modelo Educativo COMPLETO.pdf

Universidad Nacional Mayor de San Marcos - UNMSM (2016). Avances en el diseño curricular: EAP de Bibliotecología y Ciencias de la Información, Facultad de Letras y Ciencias Humanas. Lima, UNMSM. Vicerrectorado Académico. Recuperado en http://viceacademico.unmsm.edu.pe/wpcontent/uploads/2016/07/Bibliotecolog\%C3\%ADa-21.pdf

\section{Notas de los Autores}

* Alonso Estrada-Cuzcano. Docente principal, académico de la Universidad Nacional Mayor de San Marcos, Lima, Perú (UNMSM). Correo electrónico mestradac@unmsm.edu.pe

** Karen Lizeth Alfaro-Mendives. Docente asociada, académica de la Universidad Nacional Mayor de San Marcos, Lima, Perú (UNMSM). Correo electrónico kalfarom af@unmsm.edu.pe 